\title{
Dynamical Behaviour of a Tumor-Immune System with Chemotherapy and Optimal Control
}

\author{
Swarnali Sharma and G. P. Samanta \\ Department of Mathematics, Bengal Engineering and Science University, Shibpur, Howrah 711 103, India \\ Correspondence should be addressed to G. P. Samanta; g_p_samanta@yahoo.co.uk
}

Received 22 April 2013; Revised 7 June 2013; Accepted 23 June 2013

Academic Editor: Giovanni P. Galdi

Copyright (c) 2013 S. Sharma and G. P. Samanta. This is an open access article distributed under the Creative Commons Attribution License, which permits unrestricted use, distribution, and reproduction in any medium, provided the original work is properly cited.

\begin{abstract}
We have considered a tumor growth model with the effect of tumor-immune interaction and chemotherapeutic drug. We have considered two immune components-helper (resting) T-cells which stimulate CTLs and convert them into active (hunting) CTL cells and active (hunting) CTL cells which attack, destroy, or ingest the tumor cells. In our model there are four compartments, namely, tumor cells, active CTL cells, helper T-cells, and chemotherapeutic drug. We have discussed the behaviour of the solutions of our system. The dynamical behaviour of our system by analyzing the existence and stability of the system at various equilibrium points is discussed elaborately. We have set up an optimal control problem relative to the model so as to minimize the number of tumor cells and the chemotherapeutic drug administration. Here we used a quadratic control to quantify this goal and have considered the administration of chemotherapy drug as control to reduce the spread of the disease. The important mathematical findings for the dynamical behaviour of the tumor-immune model with control are also numerically verified using MATLAB. Finally, epidemiological implications of our analytical findings are addressed critically.
\end{abstract}

\section{Introduction}

It is well known that cancer is one of the greatest killer diseases in the world. Cancer, known medically as a malignant neoplasm, is characterized by an abnormal growth of cells. In cancer, cells divide and grow uncontrollably forming malignant tumors and invade nearby parts of the human body. The cancer may also spread to more distant parts of the body through the lymphatic system or bloodstream. Not all tumors are cancerous. The tumors which do not grow uncontrollably, do not invade neighbouring tissues, and do not spread throughout the body are not cancerous. There are over 200 different cancers including breast cancer, skin cancer, lung cancer, ovarian cancer, brain cancer, colon cancer, prostate cancer, and lymphoma and cancer. that afflict humans. Cancer symptoms vary widely based on the type of cancer.

Cancer immunology is the study of interactions between the immune system and cancer cells. It is also a growing field of research that aims to discover innovative cancer immunotherapies to treat and retard progression of this disease. In the last few decades, immunotherapy has become a significant part of treating several types of cancer. Human immune system is a collection of organs, special cells, and substances that help to protect from infections and some other diseases. Immune system cells and the substances they make travel through the body to protect it from pathogens (germs) causing infections. They also help to protect from cancer in some ways.

Pathogens (viruses, bacteria, and parasites) are foreign armies as they are not normally found in the body. They try to invade human body to use its resources to serve their own purposes and so they can hurt the body in the process. In fact, people often use the word foreign to describe invading germs or other substances not normally found in the body. The immune system is acting as body's defense force. It helps keep invading germs out or helps kill them if they do get into the body. The immune system basically works by keeping track of all of the substances normally found in the body, and any new substance in the body that the immune system does not recognize raises an alarm to attack it. Substances that cause an immune system response are known as antigens. The immune response can lead to destruction of anything containing the antigen, such as pathogens or cancer cells. 
Pathogens (viruses, bacteria, and parasites) have substances on their outer surfaces, such as certain proteins, that are not normally found in the human body. The immune system identifies these foreign substances as antigens. Cancer cells are also different from normal cells in the body, and they often have unusual substances on their outer surfaces that can act as antigens. But the immune system is much better at recognizing and attacking pathogens than cancer cells. Pathogens are very different from normal human cells and are often easily identified as foreign whereas cancer cells and normal cells have fewer clear differences. Due to this reason the immune system may not always recognize cancer cells as foreign. Cancer cells are less like soldiers of an invading army and more like betrayers within the ranks of the human cell population. So, the immune system's normal ability to attack cancer is limited, and this is the reason that many people with healthy immune systems still develop cancer. Sometimes the immune system recognizes the cancer cells, but the response may not be strong enough to kill them. Also, cancer cells themselves may give off substances that keep the immune system in check.

To overcome this, scientists have designed ways to help the immune system to recognize cancer cells and strengthen its response so that it will destroy them. The immune response including the recognition of cancer specific antigens is of particular interest in this field as knowledge gained drives the development of new vaccines and antibody therapies. Cellmediated immunity involves the production of cytotoxic Tlymphocytes (CTLs), activated macrophages, and release of various cytokines in response to an antigen.

A cytotoxic T-cell (CTL) is a T-lymphocyte (a type of white blood cell) that kills cancer cells, cells that are infected or cells that are damaged in other ways. Recently, increasing importance is being given to the stimulation of a CD4+ T helper cell response in cancer immunotherapy. T helper cells are a subgroup of lymphocytes, a type of white blood cell, that play an important role in the immune system, particularly in the adaptive immune system. They help in the activity of other immune cells by releasing T-cell cytokines. They assist other white blood cells in immunologic processes, including maturation of B-cells into plasma cells and memory B-cells and activation of cytotoxic T-cells (CTLs) and macrophages.

Cancer treatment includes chemotherapy, radiation therapy, immunotherapy, surgery, and monoclonal antibody therapy. The choice of therapy depends upon the location and grade of the tumor and the stage of the disease, as well as the general state of the patient. A number of experimental cancer treatments are also under development. Complete removal of the cancer without damage to the rest of the body is the goal of treatment. Sometimes this can be accomplished by surgery, but the propensity of cancers to invade adjacent tissue or to spread to distant sites by microscopic metastasis often limits its effectiveness. Chemotherapy and radiotherapy unfortunately have a negative effect on normal cells.

Chemotherapy is the treatment of cancer with one or more cytotoxic antineoplastic drugs ("chemotherapeutic agents") as part of a standardized regimen. Traditional chemotherapy drugs act by killing cells that divide rapidly, one of the main properties of the most cancer cells. This means that chemotherapy also harms cells that divide rapidly under normal circumstances: cells in the bone marrow, digestive tract, and hair follicles. This results in the most common side effects of chemotherapy: myelosuppression (decreased production of blood cells), hence, also immunosuppression, mucositis (inflammation of the lining of the digestive tract), alopecia (hair loss), and so forth. Some newer anticancer drugs (e.g., various monoclonal antibodies) are not indiscriminately cytotoxic, but rather target proteins that are abnormally expressed in cancer cells and that are essential for their growth. Such treatments are often referred to as targeted therapy (as distinct from classic chemotherapy) and are often used alongside traditional chemotherapeutic agents in antineoplastic treatment regimens.

Theoretical study of tumor-immune dynamics is very useful. Mathematical modelling in tumor growth has helped to shape our understanding of tumor-immune dynamics. Kuznetsov and Knott [1] have developed a deterministic model that describes the interplay of the cancer cells and the cytotoxic killer cells. Though they have considered only one immune cell population, they have discussed effectively the mechanisms of tumor growth, suppression, and regrowth. Kuznetsov et al. [2] presented a mathematical model of the cytotoxic T-lymphocyte response to the growth of an immunogenic tumor. Through mathematical modelling Kirschner and Panetta [3] have illustrated the dynamics between tumor cells, immune cells, and interleukin-2. Kolev [4] presented a mathematical model, showing competition between tumor cells and immune cells considering the role of antibodies. De Pillis et al. [5] presented a mathematical model on tumor growth using mixed immunotherapy and chemotherapy. De Pillis and Radunskaya [6] presented a mathematical model, showing competition between normal cells and tumor cells considering the role of chemotherapeutic drug. There are some other research works on tumorimmune dynamics [7-18]. A more common problem is found in the literature which minimizes the tumor volume at a final time subject to toxicity constraints $[19,20]$. There are some researchers who have worked on the tumor growth models with optimal control strategies $[6,20-26]$. These are very helpful to predict the most effective therapy and strategy to control the spread of diseases minimizing total drug administered. Ledzewicz and Schättler [27] presented a complete solution for a mathematical model for tumor antiangiogenesis for the problem of optimally scheduling a given amount of inhibitors in order to minimize the primary tumor volume.

In this paper, we have considered a tumor growth model together with the effect of tumor-immune interaction and chemotherapeutic drug. We have considered two immune components: (i) helper (resting) T-cells which are not able to attack and destroy tumor cells directly but release interleukin-2 which stimulates CTLs and convert them into active (hunting) CTL cells and (ii) active (hunting) CTL cells which attack, destroy, or ingest the tumor cells. In our model there are four compartments, namely, tumor cells, active CTL cells, helper T-cells, and chemotherapeutic drug. The model construction and assumptions are described in Section 2. It should be mentioned here that the recent trend 
is to incorporate a more complex biochemistry [28]. Besides tumor cells and the drug, two species of T-lymphocytes (TLs) enter the model: helper (resting) TLs and active (hunting) cytotoxic TLs, which actually destroy the tumor cells. This is an oversimplification, since in the model the two types of Tcells are supposed to interact by direct contact, ignoring the role of mediators (interleukin-2 and interferon alpha). However, dealing with a simplified model allows to give us clear insights and to get some results that may hopefully have some applicability. In Section 3, we have discussed the behaviour of the solutions of our system. The dynamical behaviour of our system by analyzing the existence and stability of the system at various equilibrium points is discussed in Section 4. In the next section, we have set up an optimal control problem relative to the model so as to minimize the number of tumor cells and the chemotherapeutic drug administration. Here we have used a quadratic control to quantify this goal and considered the administration of chemotherapeutic drug as control to reduce the spread of the disease. The quadratic control reflects the severity of the side effects of the drug imposed $[29,30]$. When chemotherapeutic drugs are administered in high dose, they are toxic to the human body, which justifies the use of quadratic control. The important mathematical findings for the dynamical behaviour of the tumor-immune model with control are also numerically verified using MATLAB in Section 6. Finally, Section 7 contains the general discussions and conclusions of the paper and epidemiological implications of our mathematical findings.

\section{Mathematical Model}

In this section we have constructed a mathematical model of tumor growth with an immune response and chemotherapy.

The model can be presented by the following set of ordinary differential equations:

$$
\begin{aligned}
& \frac{d T}{d t}=r_{1} T\left(1-p_{1} T\right)-\alpha_{1} T I_{H}-q_{1} D T, \\
& \frac{d I_{H}}{d t}=\beta I_{H} I_{R}-\alpha_{2} T I_{H}-d I_{H}-q_{2} D I_{H}, \\
& \frac{d I_{R}}{d t}=r_{2} I_{R}\left(1-p_{2} I_{R}\right)-\beta I_{H} I_{R}-q_{3} D I_{R}, \\
& \frac{d D}{d t}=u_{0}-\gamma D,
\end{aligned}
$$

with initial conditions

$$
T(0) \geq 0, \quad I_{H}(0) \geq 0, \quad I_{R}(0) \geq 0, \quad D(0) \geq 0,
$$

where $T(t), I_{H}(t), I_{R}(t)$ are the numbers of tumor cells, active CTL cells (hunting CTL cells), and helper T-cells (resting T-cells), respectively, and $D(t)$ is the density of chemotherapeutic drug at time $t$. All the model parameters $r_{1}, r_{2}, p_{1}, p_{2}, \alpha_{1} \alpha_{2}, \beta, d, \gamma, u_{0}, q_{1}, q_{2}, q_{3}$ are positive constants.
The model parameters are described as follows:

$r_{1}, r_{2}$ : per capita growth rates of tumor cells and helper (resting) T-cells, respectively;

$p_{1}, p_{2}$ : reciprocal carrying capacities for tumor cells and helper (resting) T-cells, respectively;

$\alpha_{1}, \alpha_{2}$ : rate of loss of tumor cells due to encounter with the active (hunting) CTL cells and rate of loss of active (hunting) CTL cells due to encounter with the tumor cells, respectively;

$\beta$ : rate of conversion of helper (resting) T-cells to active (hunting) CTL cells;

$d$ : per capita decay rate of active (hunting) CTL cells; $\gamma$ : per capita decay rate of the chemotherapeutic drug; $u_{0}$ : the dose of chemotherapeutic drug given;

$q_{1}, q_{2}, q_{3}$ : response coefficients to the chemotherapy drug for tumor cells, active (hunting) CTL cells, and helper (resting) T-cells, respectively.

This model involves certain assumptions which consist of the followings.

(i) The tumor cell population is assumed to grow logistically in the absence of active (hunting) CTL cells and chemotherapeutic drug.

(ii) The tumor cells are being destroyed at a rate proportional to the product of densities of tumor cells and active (hunting) CTL cells.

(iii) There is a loss in the active CTL cells due to encounters of tumor cells which is assumed to be proportional to the product of the densities of tumor cells and active CTL cells.

(iv) Helper T-cells are converted into active CTL cells either by direct contact with them or by contact with cytokines produced by the helper T-cells according to the law of mass action.

(v) The helper T-cell population is also assumed to grow logistically in the absence of active CTL cells and chemotherapeutic drug.

(vi) Chemotherapeutic drug destroys tumor cells as well as helper T-cells and active CTL cells; that is, chemotherapeutic drug has a negative effect on both tumor cells and immune cells.

\section{Behaviour of the Solutions of System (1)}

Theorem 1. Every solution of system (1) with initial conditions (2) exists in the interval $[0, \infty)$ and $T(t) \geq 0, I_{H}(t) \geq 0, I_{R}(t) \geq$ $0, D(t) \geq 0$, for all $t \geq 0$.

Proof. Since the right-hand side of system (1) is completely continuous and locally Lipschitzian on $C$, the solution $\left(T(t), I_{H}(t), I_{R}(t), D(t)\right)$ of (1) with initial conditions (2) exists 
and is unique on $[0, \xi)$, where $0<\xi \leq+\infty$ [31]. From system (1) with initial conditions (2), we have

$$
\begin{aligned}
& T(t) \\
& =T(0) \exp \left[\int_{0}^{t}\left\{r_{1}\left(1-p_{1} T(s)\right)-\alpha_{1} I_{H}(s)-q_{1} D(s)\right\} d s\right] \\
& \geq 0, \\
& \quad I_{H}(t) \\
& \quad=I_{H}(0) \exp \left[\int_{0}^{t}\left\{\beta I_{R}(s)-\alpha_{2} T(s)-d-q_{2} D(s)\right\} d s\right] \\
& \quad \geq 0, \\
& I_{R}(t) \\
& =I_{R}(0) \exp \left[\int_{0}^{t}\left\{r_{2}\left(1-p_{2} I_{R}(s)\right)-\beta I_{H}(s)-q_{3} D(s)\right\} d s\right] \\
& \geq 0, \quad D(t)=\frac{u_{0}}{\gamma}+\left[D(0)-\frac{u_{0}}{\gamma}\right] e^{-\gamma t} \geq 0,
\end{aligned}
$$

which completes the proof.

Theorem 2. $T(t), I_{R}(t), D(t)$ of system (1) subject to initial conditions (2) are bounded but $I_{H}(t)$ may be bounded under some conditions among the parameters and the bounds of $T(t)$, $I_{R}(t), D(t)$ for $t>0$.

Proof. From the first equation of system (1) it follows that

$$
\frac{d T}{d t} \leq r_{1} T\left(1-p_{1} T\right)
$$

From the standard Kamke comparison theory [15], we get

$$
\lim _{t \rightarrow \infty} \sup T(t) \leq \frac{1}{p_{1}} .
$$

Similarly, from the third equation of system (1) it follows that

$$
\frac{d I_{R}}{d t} \leq r_{2} I_{R}\left(1-p_{2} I_{R}\right)
$$

From the standard Kamke comparison theory, we get

$$
\lim _{t \rightarrow \infty} \sup I_{R}(t) \leq \frac{1}{p_{2}} .
$$

From the fourth equation of system (1), we get

$$
D(t)=\frac{u_{0}}{\gamma}+\left[D(0)-\frac{u_{0}}{\gamma}\right] e^{-\gamma t}
$$

Therefore,

$$
\lim _{t \rightarrow \infty} \sup D(t) \leq \frac{u_{0}}{\gamma}
$$

Now, from the second equation of system (1) it follows that

$$
\begin{aligned}
& I_{H}(t) \\
& =I_{H}(0) \exp \left[\int_{0}^{t}\left\{\beta I_{R}(\omega)-\alpha_{2} T(\omega)-d-q_{2} D(\omega)\right\} d \omega\right] .
\end{aligned}
$$

Let us assume that $\sup (t)=T_{s}, \inf T(t)=T_{i}, \sup I_{R}(t)=I_{R_{s}}$, $\inf I_{R}(t)=I_{R_{i}}$, sup $D(t)=D_{s}$, inf $D(t)=D_{i}$ as $T(t), I_{R}(t)$, and $D(t)$ are bounded.

Now,

$$
\begin{aligned}
& \beta I_{R}(\omega)-\alpha_{2} T(\omega)-d-q_{2} D(\omega) \\
& \quad \leq \beta I_{R_{s}}-\alpha_{2} T_{i}-d-q_{2} D_{i} \\
& \quad \leq-A \text { (say), } \quad A>0 \text { (by assumption). }
\end{aligned}
$$

Therefore,

$$
\begin{aligned}
I_{H}(t) & \leq I_{H}(0) \exp \left[\int_{0}^{t}(-A) d \omega\right] \\
& =I_{H}(0) e^{-A t} .
\end{aligned}
$$

So,

$$
\lim _{t \rightarrow \infty} \sup I_{H}(t)=0 \quad \text { as } A>0
$$

Therefore, we can conclude that $I_{H}(t)$ may be bounded under some conditions among the parameters and the bounds of $T(t), I_{R}(t), D(t)$ for $t>0$.

Hence, the theorem.

\section{Equilibrium Points: Their Existence and Stability}

In this section we will study the existence and stability behaviour of the system (1) at various equilibrium points. The equilibrium points of the system (1) are

(i) trivial equilibrium: $E_{0}\left(0,0,0, u_{0} / \gamma\right)$,

(ii) tumor persistent equilibrium:
(a) $E_{1}(\bar{T}, 0,0, \bar{D})$, where $\bar{T}=\left(r_{1} \gamma-q_{1} u_{0}\right) / p_{1} r_{1} \gamma$, $\bar{D}=u_{0} / \gamma$
(b) $E_{2}\left(\widehat{T}, 0, \widehat{I}_{R}, \widehat{D}\right)$, where $\widehat{T}=\left(r_{1} \gamma-q_{1} u_{0}\right) / p_{1} r_{1} \gamma$, $\widehat{I}_{R}=\left(r_{2} \gamma-q_{3} u_{0}\right) / p_{2} r_{2} \gamma, \widehat{D}=u_{0} / \gamma$

(iii) tumor free equilibrium:

(a) $E_{3}\left(0,0, \widetilde{I}_{R}, \widetilde{D}\right)$, where $\widetilde{I}_{R}=\left(r_{2} \gamma-q_{3} u_{0}\right) / p_{2} r_{2} \gamma$, $\widetilde{D}=u_{0} / \gamma$

(b) $E_{4}\left(0, \check{I}_{H}, \check{I}_{R}, \check{D}\right)$, where $\check{I}_{H}=\left(1 / \beta^{2} \gamma\right)\left(r_{2} \beta \gamma-\right.$ $\left.d p_{2} r_{2} \gamma-q_{3} \beta u_{0}-p_{2} q_{2} r_{2} u_{0}\right), \check{I}_{R}=\left(q_{2} u_{0}+d \gamma\right) / \beta \gamma$, $\check{D}=u_{0} / \gamma$; 
(iv) coexisting equilibrium: $E^{*}\left(T^{*}, I_{H}^{*}, I_{R}^{*}, D^{*}\right)$, where

$$
\begin{aligned}
T^{*}= & \left(p_{2} r_{2} \alpha_{1}\left(q_{2} u_{0}+d \gamma\right)\right. \\
& \left.-\beta\left(r_{2} \alpha_{1} \gamma+q_{1} u_{0} \beta-q_{3} \alpha_{1} u_{0}-r_{1} \beta \gamma\right)\right) \\
& \times\left(\left(p_{1} r_{1} \beta^{2}-p_{2} r_{2} \alpha_{1} \alpha_{2}\right) \gamma\right)^{-1}, \\
I_{H}^{*}= & \left(p_{2} r_{2} \alpha_{2}\left(q_{1} u_{0}-r_{1} \gamma\right)\right. \\
& \left.\quad-p_{1} r_{1}\left(p_{2} q_{2} r_{2} u_{0}+q_{3} \beta u_{0}+d p_{2} r_{2} \gamma-r_{2} \beta \gamma\right)\right) \\
& \times\left(\left(p_{1} r_{1} \beta^{2}-p_{2} r_{2} \alpha_{1} \alpha_{2}\right) \gamma\right)^{-1}, \\
I_{R}^{*}= & \left(q_{3} \alpha_{1} \alpha_{2} u_{0}+p_{1} r_{1} \beta\left(q_{2} u_{0}+d \gamma\right)\right. \\
& \left.\quad-\alpha_{2}\left(q_{1} \beta u_{0}+r_{2} \alpha_{1} \gamma-r_{1} \beta \gamma\right)\right) \\
& \times\left(\left(p_{1} r_{1} \beta^{2}-p_{2} r_{2} \alpha_{1} \alpha_{2}\right) \gamma\right)^{-1}, \\
D^{*}= & \frac{u_{0}}{\gamma} .
\end{aligned}
$$

Trivial Equilibrium. Now, the variational matrix of system (1) at $E_{0}(0,0,0,0)$ is given by

$$
\begin{aligned}
& V\left(E_{0}\right) \\
& =\left(\begin{array}{cccc}
r_{1}-q_{1} \frac{u_{0}}{\gamma} & 0 & 0 & 0 \\
0 & -d-q_{2} \frac{u_{0}}{\gamma} & 0 & 0 \\
0 & 0 & r_{2}-q_{3} \frac{u_{0}}{\gamma} & 0 \\
0 & 0 & 0 & -\gamma
\end{array}\right) .
\end{aligned}
$$

Therefore, eigenvalues of the characteristic equation of $V\left(E_{0}\right)$ are $\lambda_{1}=\left(r_{1}-q_{1}\left(u_{0} / \gamma\right)\right), \lambda_{2}=\left(-d-q_{2}\left(u_{0} / \gamma\right)\right), \lambda_{3}=\left(r_{2}-\right.$ $\left.q_{3}\left(u_{0} / \gamma\right)\right), \lambda_{4}=-\gamma$. It is clear that $\lambda_{2}, \lambda_{4}$ are negative. Now, $E_{0}$ is stable if $\lambda_{1}<0$ and $\lambda_{3}<0$; that is, $r_{1}-q_{1}\left(u_{0} / \gamma\right)<0$ and $r_{2}-q_{3}\left(u_{0} / \gamma\right)<0$, which implies that $u_{0}>r_{1} \gamma / q_{1}$ and $u_{0}>r_{2} \gamma / q_{3}$.

\section{Tumor Persistent Equilibrium. Consider the following}

(i) $E_{1}(\bar{T}, 0,0, \bar{D})$ exists only when $r_{1} \gamma-q_{1} u_{0}>0$; that is, $u_{0}<r_{1} \gamma / q_{1}$.

Now, the variational matrix of system $(1)$ at $E_{1}(\bar{T}, 0,0, \bar{D})$ is given by

$$
V\left(E_{1}\right)=\left(\begin{array}{cccc}
-r_{1} p_{1} \bar{T} & -\alpha_{1} \bar{T} & 0 & -q_{1} \bar{T} \\
0 & -\alpha_{2} \bar{T}-d-q_{2} \bar{D} & 0 & 0 \\
0 & 0 & r_{2}-q_{3} \bar{D} & 0 \\
0 & 0 & 0 & -\gamma
\end{array}\right) .
$$

Therefore, eigenvalues of the characteristic equation of $V\left(E_{1}\right)$ are $\lambda_{1}=\left(-r_{1} p_{1} \bar{T}\right), \lambda_{2}=\left(-\alpha_{2} \bar{T}-d-q_{2} \bar{D}\right), \lambda_{3}=\left(r_{2}-q_{3} \bar{D}\right)$, $\lambda_{4}=-\gamma$. It is clear that $\lambda_{1}, \lambda_{2}, \lambda_{4}$ are negative. Now, $E_{1}$ is stable if $\lambda_{3}<0$; that is, $r_{2}-q_{3} \bar{D}<0$ which implies that $u_{0}>r_{2} \gamma / q_{3}$. (ii) $E_{2}\left(\widehat{T}, 0, \widehat{I}_{R}, \widehat{D}\right)$ exists only when $r_{1} \gamma-q_{1} u_{0}>0$ and $r_{2} \gamma-q_{3} u_{0}>0$; that is, $u_{0}<r_{1} \gamma / q_{1}$ and $u_{0}<r_{2} \gamma / q_{3}$.

Now, the variational matrix of system $(1)$ at $E_{2}\left(\widehat{T}, 0, \widehat{I}_{R}, \widehat{D}\right)$ is given by

$$
\begin{aligned}
& V\left(E_{2}\right) \\
& =\left(\begin{array}{cccc}
-r_{1} p_{1} \widehat{T} & -\alpha_{1} \widehat{T} & 0 & -q_{1} \widehat{T} \\
0 & \beta \widehat{I}_{R}-\alpha_{2} \widehat{T}-d-q_{2} \widehat{D} & 0 & 0 \\
0 & -\beta \widehat{I}_{R} & -r_{2} p_{2} \widehat{I}_{R} & -q_{3} \widehat{I}_{R} \\
0 & 0 & 0 & -\gamma
\end{array}\right) .
\end{aligned}
$$

Therefore, eigenvalues of the characteristic equation of $V\left(E_{2}\right)$ are $\lambda_{1}=-r_{1} p_{1} \widehat{T}, \lambda_{2}=\left(\beta \widehat{I}_{R}-\alpha_{2} \widehat{T}-d-q_{2} \widehat{D}\right), \lambda_{3}=$ $-r_{2} p_{2} \widehat{I}_{R}, \lambda_{4}=-\gamma$. It is clear that $\lambda_{1}, \lambda_{3}, \lambda_{4}$ are negative. Now, $E_{2}$ is stable if $\lambda_{2}<0$; that is, $\beta \widehat{I}_{R}-\alpha_{2} \widehat{T}-d-q_{2} \widehat{D}<0$ which implies that $\beta<\left(\alpha_{2} \widehat{T}+d+q_{2} \widehat{D}\right) / \widehat{I}_{R}$. result.

From the previous discussion we come to the following

Theorem 3. The tumor persistent equilibrium $E_{1}$ of the system (1) exists and is locally asymptotically stable if

$$
\frac{r_{2} \gamma}{q_{3}}<u_{0}<\frac{r_{1} \gamma}{q_{1}}
$$

and another tumor free equilibrium $E_{2}$ of the system (1) exists and is locally asymptotically stable if

$$
u_{0}<\frac{r_{1} \gamma}{q_{1}}, \quad u_{0}<\frac{r_{2} \gamma}{q_{3}}, \quad \beta<\frac{\alpha_{1} \widehat{T}+d+q_{2} \widehat{D}}{\widehat{I}_{R}} .
$$

Tumor Free Equilibrium. Consider the following

(i) $E_{3}\left(0,0, \widetilde{I}_{R}, \widetilde{D}\right)$ exists only when $r_{2} \gamma-q_{3} u_{0}>0$; that is, $u_{0}<r_{2} \gamma / q_{3}$

Now, the variational matrix of system $(1)$ at $E_{3}\left(0,0, \widetilde{I}_{R}, \widetilde{D}\right)$ is given by

$$
V\left(E_{3}\right)=\left(\begin{array}{cccc}
r_{1}-q_{1} \widetilde{D} & 0 & 0 & 0 \\
0 & \beta \widetilde{I}_{R}-d-q_{2} \widetilde{D} & 0 & 0 \\
0 & -\beta \widetilde{I}_{R} & -r_{2} p_{2} \widetilde{I}_{R} & -q_{3} \widetilde{I}_{R} \\
0 & 0 & 0 & -\gamma
\end{array}\right)
$$

Therefore, eigenvalues of the characteristic equation of $V\left(E_{3}\right)$ are $\lambda_{1}=\left(r_{1}-q_{1} \widetilde{D}\right), \lambda_{2}=\left(\beta \widetilde{I}_{R}-d-q_{2} \widetilde{D}\right), \lambda_{3}=-r_{2} p_{2} \widetilde{I}_{R}$, $\lambda_{4}=-\gamma$. It is clear that $\lambda_{3}, \lambda_{4}$ are negative. Now, $E_{3}$ is stable if $\lambda_{1}<0$ and $\lambda_{2}<0$; that is, $r_{1}-q_{1} \widetilde{D}<0$ and $\beta \widetilde{I}_{R}-d-q_{2} \widetilde{D}<0$, which implies that $u_{0}>r_{1} \gamma / q_{1}$ and $\beta<\left(d \gamma+q_{2} u_{0}\right) / \gamma \widetilde{I}_{R}$.

(ii) $E_{4}\left(0, \check{I}_{H}, \check{I}_{R}, \check{D}\right)$ exists only when $u_{0}<\left(\beta-d p_{2}\right) r_{2} \gamma /$ $\left(q_{3} \beta+p_{2} q_{2} r_{2}\right)$. 
Now, the variational matrix of system $(1)$ at $E_{4}\left(0, \check{I}_{H}, \check{I}_{R}, \check{D}\right)$ is given by

$$
V\left(E_{4}\right)=\left(\begin{array}{cccc}
r_{1}-\alpha_{1} \check{I}_{H}-q_{1} \check{D} & 0 & 0 & 0 \\
-\alpha_{2} \widehat{I}_{H} & 0 & \beta \check{I}_{H} & -q_{2} \check{I}_{H} \\
0 & -\beta \check{I}_{R} & -r_{2} p_{2} \check{I}_{R} & -q_{3} \check{I}_{R} \\
0 & 0 & 0 & -\gamma
\end{array}\right) .
$$

Therefore, eigenvalues of the characteristic equation of $V\left(E_{4}\right)$ are $\left(r_{1}-\alpha_{1} \check{I}_{H}-q_{1} \check{D}\right),-\gamma$ and the solution of the quadratic equation

$$
P(\lambda)=\lambda^{2}+m_{1} \lambda+m_{2}=0
$$

where

$$
m_{1}=r_{2} p_{2} \check{I}_{R}>0, \quad m_{2}=\beta^{2} \check{I}_{R} \check{I}_{H}>0 .
$$

Now, it is easily noted that as $m_{1}>0$ and $m_{2}>0, P(\lambda)$ has negative real roots. Therefore, $E_{4}$ is stable only when $r_{1}-$ $\alpha_{1} \check{I}_{H}-q_{1} \check{D}<0$; that is, $r_{1}<\alpha_{1} \check{I}_{H}+q_{1} \check{D}$.

From the previous discussion we come to the following result.

Theorem 4. The tumor free equilibrium $E_{3}$ of the system (1) exists and is locally asymptotically stable if

$$
\frac{r_{1} \gamma}{q_{1}}<u_{0}<\frac{r_{2} \gamma}{q_{3}}, \quad \beta<\frac{d \gamma+q_{2} u_{0}}{\gamma \widetilde{I}_{R}},
$$

and another tumor free equilibrium $E_{4}$ of the system (1) exists and is locally asymptotically stable if

$$
u_{0}<\frac{\left(\beta-d p_{1}\right) r_{2} \gamma}{q_{3} \beta+p_{2} q_{2} r_{2}}, \quad r_{1}<\alpha_{1} \check{I}_{H}+q_{1} \check{D} .
$$

Coexisting Equilibrium. $E^{*}\left(T^{*}, I_{H}^{*}, I_{R}^{*}, D^{*}\right)$ exists when

(i) $p_{2} r_{2} \alpha_{1} \alpha_{2}-p_{1} r_{1} \beta^{2}>0, \beta\left(-q_{3} \alpha_{1} u_{0}+q_{1} \beta u_{0}+r_{2} \alpha_{1} \gamma-\right.$ $\left.r_{1} \beta \gamma\right)-p_{2} r_{2} \alpha_{1}\left(q_{2} u_{0}+d \gamma\right)>0, p_{2} r_{2} \alpha_{2}\left(-q_{1} u_{0}+\right.$ $\left.r_{1} \gamma\right)+p_{1} r_{1}\left(p_{2} q_{2} r_{2} u_{0}+q_{3} \beta u_{0}+d p_{2} r_{2} \gamma-r_{2} \beta \gamma\right)>0$, $\alpha_{2}\left(q_{1} \beta u_{0}+r_{2} \alpha_{2} \gamma-r_{1} \beta \gamma\right)-q_{3} \alpha_{1} \alpha_{2} u_{0}-p_{1} r_{1} \beta\left(q_{2} u_{0}+\right.$ $d \gamma)>0$

or

(ii) $p_{2} r_{2} \alpha_{1} \alpha_{2}-p_{1} r_{1} \beta^{2}<0, \beta\left(-q_{3} \alpha_{1} u_{0}+q_{1} \beta u_{0}+r_{2} \alpha_{1} \gamma-\right.$ $\left.r_{1} \beta \gamma\right)-p_{2} r_{2} \alpha_{1}\left(q_{2} u_{0}+d \gamma\right)<0, p_{2} r_{2} \alpha_{2}\left(-q_{1} u_{0}+\right.$ $\left.r_{1} \gamma\right)+p_{1} r_{1}\left(p_{2} q_{2} r_{2} u_{0}+q_{3} \beta u_{0}+d p_{2} r_{2} \gamma-r_{2} \beta \gamma\right)<0$, $\alpha_{2}\left(q_{1} \beta u_{0}+r_{2} \alpha_{2} \gamma-r_{1} \beta \gamma\right)-q_{3} \alpha_{1} \alpha_{2} u_{0}-p_{1} r_{1} \beta\left(q_{2} u_{0}+\right.$ $d \gamma)<0$.

Now, the variational matrix of system (1) at $E^{*}\left(T^{*}\right.$, $\left.I_{H}^{*}, I_{R}^{*}, D^{*}\right)$ is given by

$$
V\left(E^{*}\right)=\left(\begin{array}{cccc}
-r_{1} p_{1} T^{*} & -\alpha_{1} T^{*} & 0 & -q_{1} T^{*} \\
-\alpha_{2} I_{H}^{*} & 0 & \beta I_{H}^{*} & -q_{2} I_{H}^{*} \\
0 & -\beta I_{R}^{*} & -r_{2} p_{2} I_{R}^{*} & -q_{3} I_{R}^{*} \\
0 & 0 & 0 & -\gamma
\end{array}\right) .
$$

Therefore, eigenvalues of the characteristic equation of $V\left(E^{*}\right)$ are $-\gamma$ and the solution of the following equation,

$$
\lambda^{3}+A_{1} \lambda^{2}+A_{2} \lambda+A_{3}=0
$$

where

$$
\begin{gathered}
A_{1}=-a_{11}-a_{33}, \\
A_{2}=a_{11} a_{33}-a_{12} a_{21}-a_{23} a_{32}, \\
A_{3}=a_{11} a_{23} a_{32}+a_{12} a_{21} a_{33}, \\
a_{11}=-r_{1} p_{1} T^{*}, \quad a_{12}=-\alpha_{1} T^{*}, \quad a_{14}=-q_{1} T^{*}, \\
a_{21}=-\alpha_{2} I_{H}^{*}, \quad a_{23}=\beta I_{H}^{*}, \quad a_{24}=-q_{2} I_{H}^{*}, \\
a_{32}=-\beta I_{R}^{*}, \quad a_{33}=-r_{2} p_{2} I_{R}^{*}, \quad a_{34}=-q_{3} I_{R}^{*}, \\
a_{44}=-\gamma .
\end{gathered}
$$

By the Routh-Hurwitz criterion [32], it follows that the roots of (27) have negative real part if and only if

$$
A_{1}>0, \quad A_{3}>0, \quad A_{1} A_{2}-A_{3}>0 .
$$

Now, it is easy to be noted that $A_{1}=-a_{11}-a_{33}=r_{1} p_{1} T^{*}+$ $r_{2} p_{2} I_{R}^{*}>0$ and $A_{3}=a_{11} a_{23} a_{32}+a_{12} a_{21} a_{33}=\left(r_{1} p_{1} \beta^{2}-\right.$ $\left.\alpha_{1} \alpha_{2} r_{2} p_{2}\right) T^{*} I_{R}^{*} I_{H}^{*}>0$ if $r_{1} p_{1} \beta^{2}-\alpha_{1} \alpha_{2} r_{2} p_{2}>0$. Now, if

$$
A_{1} A_{2}-A_{3}>0
$$

then $E^{*}$ will be locally asymptotically stable. So, we came to the following result.

Theorem 5. The coexisting equilibrium $E^{*}$ of the system (1) exists and is locally asymptotically stable if

(i) $p_{1} r_{1} \beta^{2}-p_{2} r_{2} \alpha_{1} \alpha_{2}>0$,

(ii) $p_{2} r_{2} \alpha_{1}\left(q_{2} u_{0}+d \gamma\right)-\beta\left(-q_{3} \alpha_{1} u_{0}+q_{1} \beta u_{0}+r_{2} \alpha_{1} \gamma-\right.$ $\left.r_{1} \beta \gamma\right)>0$,

(iii) $p_{2} r_{2} \alpha_{2}\left(q_{1} u_{0}-r_{1} \gamma\right)-p_{1} r_{1}\left(p_{2} q_{2} r_{2} u_{0}+q_{3} \beta u_{0}+d p_{2} r_{2} \gamma-\right.$ $\left.r_{2} \beta \gamma\right)>0$,

(iv) $q_{3} \alpha_{1} \alpha_{2} u_{0}+p_{1} r_{1} \beta\left(q_{2} u_{0}+d \gamma\right)-\alpha_{2}\left(q_{1} \beta u_{0}+r_{2} \alpha_{1} \gamma-\right.$ $\left.r_{1} \beta \gamma\right)>0$,

(v) condition (30) is satisfied.

Observations. The equilibrium points for the system without chemotherapeutic drug are

(1) $\bar{E}_{0}(0,0,0)$,

(2) $\bar{E}_{1}=\left(1 / p_{1}, 0,0\right)$,

(3) $\bar{E}_{2}=\left(1 / p_{1}, 0,1 / p_{2}\right)$,

(4) $\bar{E}_{3}=\left(0,0,1 / p_{2}\right)$, 
TABLE 1

\begin{tabular}{lc}
\hline Parameter & Estimated value \\
\hline$r_{1}$ & $0.44 /$ day \\
$r_{2}$ & $0.0245 /$ day \\
$\alpha_{1}$ & $1.101 \times 10^{-7} /$ cells/day \\
$\alpha_{2}$ & $3.422 \times 10^{-10} /$ cells/day \\
$\beta$ & $6.2 \times 10^{-9} /$ cells/day \\
$\gamma$ & $0.01 /$ day \\
$p_{1}$ & $5 \times 10^{-9} /$ cells \\
$p_{2}$ & $1 \times 10^{-10} /$ cells \\
$q_{1}$ & $0.08 /$ day \\
$q_{2}$ & $2 \times 10^{-11} /$ day \\
$q_{3}$ & $1 \times 10^{-5} /$ day \\
$d$ & $0.0412 /$ day \\
\hline
\end{tabular}

(5) $\bar{E}_{4}=\left(0, r_{2}\left(\beta-d p_{2}\right) / \beta^{2}, d / \beta\right)$,

(6) $\bar{E}^{*}=\left(\bar{T}^{*}, \bar{I}_{H}^{*}, \bar{I}_{R}^{*}\right)$, where

$$
\begin{gathered}
\bar{T}^{*}=\frac{\alpha_{1} d p_{2} r_{2}+\beta\left(r_{1} \beta-\alpha_{1} r_{2}\right)}{\left(p_{1} r_{1} \beta^{2}-p_{2} r_{2} \alpha_{1} \alpha_{2}\right)}, \\
\bar{I}_{H}^{*}=\frac{r_{1} r_{2}\left\{p_{1} \beta-p_{2}\left(d p_{1}+\alpha_{2}\right)\right\}}{\left(p_{1} r_{1} \beta^{2}-p_{2} r_{2} \alpha_{1} \alpha_{2}\right)}, \\
\bar{I}_{R}^{*}=\frac{\beta r_{1}\left(d p_{1}+\alpha_{2}\right)-r_{2} \alpha_{1} \alpha_{2}}{\left(p_{1} r_{1} \beta^{2}-p_{2} r_{2} \alpha_{1} \alpha_{2}\right)} .
\end{gathered}
$$

Using the parameter values given in Table $1[2,33-36]$ we get that the equilibrium points $E_{1}, E_{2}, E_{3}$ become unstable and $E^{*}$ does not exist. The only stable equilibrium point is $E_{4}$. Here we are interested in the tumor free equilibrium $E_{4}$. The tumor free equilibrium $E_{4}\left(0, \check{I}_{H}, \check{I}_{R}, \check{D}\right)$ becomes locally asymptotically stable when $r_{1}<\alpha_{1} \check{I}_{H}+q_{1} \check{D}$, where the same tumor free equilibrium without drug, that is, $\bar{E}_{4}\left(0, I_{H}, I_{R}\right)$, is locally asymptotically stable when $r_{1}<\alpha_{1} I_{H}$.

Using the parameter values given in Table 1, we get

$$
\begin{gathered}
E_{4}\left(0, \check{I}_{H} \check{I}_{R}, \check{D}\right) \\
=E_{4}\left(0,3.9485 \times 10^{6}, 6.64516 \times 10^{6}, 0.3\right) \\
\left(\text { assuming } u_{0}=0.003\right), \\
\bar{E}_{4}\left(0, I_{H}, I_{R}\right)=\bar{E}_{4}\left(0,3.94899 \times 10^{6}, 6.64516 \times 10^{6}\right), \\
r_{1}=0.44, \quad \alpha_{1} \check{I}_{H}+q_{1} \check{D}=0.45872985, \\
\alpha_{1} I_{H}=0.434783799 .
\end{gathered}
$$

Therefore, we get

$$
\alpha_{1} I_{H}<r_{1}<\alpha_{1} \check{I}_{H}+q_{1} \check{D},
$$

which implies that using the parameter values given in Table 1 the tumor free equilibrium with chemotherapeutic drug, that is, $E_{4}$ becomes locally asymptotically stable whereas the tumor free equilibrium without drug, that is, $\bar{E}_{4}$ becomes unstable (using the same parameter values). This reasonably implies that if the chemotherapeutic drug is turned on then the tumor free equilibrium becomes stable where it is unstable without drug.

\section{Epidemic Model with Control}

In the context of mathematical modelling in cancer growth with chemotherapy, it is essential to frame an optimal control problem so that the total amount of drug used is minimized. This is done because of the implicit understanding that chemotherapy has damaging side effects. We have considered the tumor growth model (1). Now let us assume that the dose of chemotherapeutic drug is given as a function of time denoted by $u(t)$. We will use $u(t)$ as a control to decrease the tumor burden minimizing total drug administered. Here we consider a quadratic control to quantify this goal. Therefore, our tumor growth model with control becomes

$$
\begin{aligned}
\frac{d T}{d t} & =r_{1} T\left(1-p_{1} T\right)-\alpha_{1} T I_{H}-q_{1} D T, \\
\frac{d I_{H}}{d t} & =\beta I_{H} I_{R}-\alpha_{2} T I_{H}-d I_{H}-q_{2} D I_{H}, \\
\frac{d I_{R}}{d t} & =r_{2} I_{R}\left(1-p_{2} I_{R}\right)-\beta I_{H} I_{R}-q_{3} D I_{R}, \\
\frac{d D}{d t} & =u(t)-\gamma D,
\end{aligned}
$$

satisfying

$$
\begin{aligned}
T(0)=T_{0}, & I_{H}(0)=I_{H_{0}}, \quad I_{R}(0)=I_{R_{0}}, \\
D(0) & =D_{0} .
\end{aligned}
$$

The objective functional $[16,29,30,37-40]$ is defined as

$$
J(u(t))=\int_{0}^{t_{f}}\left[B_{1} T+\frac{1}{2} B_{2} u^{2}\right] d t,
$$

where $B_{1}, B_{2}$ are positive constants representing the weights of the terms. The first term represents number of cancer cells and the second term represents harmful effects of drug on body. The square of the control variable $\left(u^{2}(t)\right)$ reflects the severity of the side effects of the drug imposed $[29,30]$. When chemotherapeutic drugs are administered in high dose, they are toxic to the human body, which justifies the quadratic terms in the functional. Here the functional given in (36) should be minimized. So, we seek an optimal control $u^{*}$ such that

$$
J\left(u^{*}\right)=\min \{J(u): u \in U\},
$$

where $U=\left\{u: u\right.$ is measurable, $\left.0 \leq u(t) \leq 1, t \in\left[0, t_{f}\right]\right\}$ is the admissible control set.

5.1. Existence of an Optimal Control. Consider the following.

Theorem 6. Given the objective functional

$$
J(u(t))=\int_{0}^{t_{f}}\left[B_{1} T+\frac{1}{2} B_{2} u^{2}\right] d t,
$$

where $U=\left\{u: u\right.$ is measurable, $\left.0 \leq u(t) \leq 1, t \in\left[0, t_{f}\right]\right\}$ subject to the system (34) with (35), then there exists an optimal control $u^{*}$ such that $J\left(u^{*}\right)=\min \{J(u): u \in U\}$, if the following conditions [41] are satisfied. 
(1) The class of all initial conditions with a control $u(t)$ in the admissible control set along with each state equation being satisfied is not empty.

(2) The admissible control set $U$ is closed and convex.

(3) Each right-hand side of the state system (34) is continuous and is bounded above by a sum of the bounded control and the state and can be written as a linear function of $u$ with coefficients depending on time and the state.

(4) The integrand of $J(u)$ is convex on $U$ and is bounded below by $c_{1} u^{2}-c_{2}$ with $c_{1}, c_{2}>0$.

Proof. In order to verify the first condition, we use a result by Lukes ([42], Theorem 9.2.1) for the system (34) with bounded coefficients. The control set $U$ is convex and closed by definition, which gives the condition (2). The right-hand side of the state system (34) satisfies condition (3) as the state solutions are a priori bounded.

We let $\vec{\delta}(t, \vec{X})$ be the right-hand side of the system (34) without $u(t)$ and let

$$
\vec{f}(t, \vec{X}, u)=\vec{\delta}(t, \vec{X})+\left(\begin{array}{c}
0 \\
0 \\
0 \\
u
\end{array}\right)
$$

with

$$
\vec{X}=\left(\begin{array}{c}
T \\
I_{H} \\
I_{R} \\
D
\end{array}\right)
$$

Using the boundedness of the solutions, we see that

$$
\begin{aligned}
|\vec{f}(t, \vec{X}, u)| & \leq\left|\left(\begin{array}{cccc}
r_{1} & 0 & 0 & 0 \\
0 & \beta & 0 & 0 \\
0 & 0 & r_{2} & 0 \\
0 & 0 & 0 & -\gamma
\end{array}\right)\left(\begin{array}{c}
T \\
I_{H} \\
I_{R} \\
D
\end{array}\right)\right|+\left|\left(\begin{array}{c}
0 \\
0 \\
0 \\
u
\end{array}\right)\right| \\
& \leq C_{1}(|\vec{X}|+|u|),
\end{aligned}
$$

where $C_{1}$ depends on the coefficients of the system.

For the fourth condition we need to show that

$$
J((1-p) u+p v) \leq(1-p) J(u)+p J(v),
$$

where $u, v$ are distinct elements of $U$ and $0 \leq p \leq 1$. Now,

$$
\begin{aligned}
J( & (1-p) u+p v)-[(1-p) J(u)+p J(v)] \\
= & B_{1} T(t)+\frac{B_{2}}{2}\{(1-p) u+p v\}^{2} \\
& -\left[(1-p)\left\{B_{1} T(t)+\frac{B_{2}}{2} u^{2}\right\}+p\left\{B_{1} T(t)+\frac{B_{2}}{2} v^{2}\right\}\right] \\
= & \frac{B_{2}}{2}\left(p^{2}-p\right)(u-v)^{2} .
\end{aligned}
$$

Since $p \in[0,1]$ implies that $\left(p^{2}-p\right) \leq 0$ and $(u-v)^{2}>0$, the expression $\left(B_{2} / 2\right)\left(p^{2}-p\right)(u-v)^{2} \leq 0$, which implies that

$$
J((1-p) u+p v) \leq(1-p) J(u)+p J(v) .
$$

Lastly,

$$
\begin{aligned}
& B_{1} T(t)+\frac{1}{2} B_{2} u^{2}(t) \\
& \quad \geq \frac{B_{2}}{2} u^{2}(t) \geq \frac{B_{2}}{2} u^{2}(t)-c_{2} \geq c_{1} u^{2}(t)-c_{2},
\end{aligned}
$$

which gives $c_{1} u^{2}(t)-c_{2}$ as a lower bound of $J(u)$.

Therefore, we can conclude that there exists an optimal control $u^{*}$ such that

$$
J\left(u^{*}\right)=\min \{J(u): u \in U\} .
$$

5.2. Characterization of the Optimal Control. In order to derive the necessary conditions for the optimal control, Pontryagin's Maximum Principle [43] is invoked.

The Hamiltonian is defined as follows:

$$
\begin{aligned}
H= & \left(B_{1} T+\frac{1}{2} B_{2} u_{1}^{2}\right)+\lambda_{1}\left[r_{1} T\left(1-p_{T}\right)-\alpha_{1} T I_{H}-q_{1} D T\right] \\
& +\lambda_{2}\left[\beta I_{H} I_{R}-\alpha_{2} T I_{H}-d I_{H}-q_{2} D I_{H}\right] \\
& +\lambda_{3}\left[r_{2} I_{R}\left(1-p_{2} I_{R}\right)-\beta I_{H} I_{R}-q_{3} D I_{R}\right]+\lambda_{4}[u-\gamma D],
\end{aligned}
$$

where $\lambda_{i}(t), i=1,2,3,4$, are the adjoint functions to be determined suitably.

The form of the adjoint equations and transversality conditions are standard results from Pontryagin's Maximum Principle [43]. The adjoint system can be obtained as follows:

$$
\begin{aligned}
\frac{d \lambda_{1}}{d t} & =-\left(\frac{\partial H}{\partial T}\right) \\
& =\left(2 r_{1} p_{1} T+\alpha_{1} I_{H}+q_{1} D-r_{1}\right) \lambda_{1}+\lambda_{2} \alpha_{2} I_{H}-B_{1} \\
\frac{d \lambda_{2}}{d t} & =-\left(\frac{\partial H}{\partial I_{H}}\right) \\
& =\left(\alpha_{2} T+d+q_{2} D-\beta I_{R}\right) \lambda_{2}+\lambda_{1} \alpha_{1} T+\lambda_{3} \beta I_{R} \\
\frac{d \lambda_{3}}{d t} & =-\left(\frac{\partial H}{\partial I_{R}}\right)=\left(2 r_{2} p_{2} I_{R}+\beta I_{H}+q_{3} D\right) \lambda_{3}-\lambda_{2} \beta I_{H} \\
\frac{d \lambda_{4}}{d t} & =-\left(\frac{\partial H}{\partial D}\right)=\lambda_{1} q_{1} T+\lambda_{2} q_{2} I_{H}+\lambda_{3} q_{3} I_{R}+\lambda_{4} \gamma
\end{aligned}
$$

The transversality conditions (or boundary conditions) are

$$
\lambda_{i}\left(t_{f}\right)=0, \quad \text { for } i=1,2,3,4 .
$$

By the optimality condition, we have

$$
\begin{aligned}
\frac{\partial H}{\partial u} & =B_{2} u^{*}+\lambda_{4}=0 \quad \text { at } u_{1}=u_{1}^{*}(t) \\
& \Longrightarrow u^{*}(t)=-\frac{\lambda_{4}}{B_{2}} .
\end{aligned}
$$


By using the bounds for the control $u_{1}(t)$, we get

$$
u^{*}= \begin{cases}-\frac{\lambda_{4}}{B_{2}}, & \text { if } 0 \leq-\frac{\lambda_{4}}{B_{2}} \leq 1, \\ 0, & \text { if }-\frac{\lambda_{4}}{B_{2}} \leq 0, \\ 1, & \text { if }-\frac{\lambda_{4}}{B_{2}} \geq 1 .\end{cases}
$$

In compact notation,

$$
u^{*}=\min \left\{\max \left\{0,-\frac{\lambda_{4}}{B_{2}}\right\}, 1\right\}
$$

Using (52) we obtain the following optimal system:

$$
\begin{aligned}
& \frac{d T}{d t}=r_{1} T\left(1-p_{1} T\right)-\alpha_{1} T I_{H}-q_{1} D T \\
& \frac{d I_{H}}{d t}=\beta I_{H} I_{R}-\alpha_{2} T I_{H}-d I_{H}-q_{2} D I_{H} \\
& \frac{d I_{R}}{d t}=r_{2} I_{R}\left(1-p_{2} I_{R}\right)-\beta I_{H} I_{R}-q_{3} D I_{R} \\
& \frac{d D}{d t}=\min \left\{\max \left\{0,-\frac{\lambda_{4}}{B_{2}}\right\}, 1\right\}-\gamma D \\
& \frac{d \lambda_{1}}{d t}=\left(2 r_{1} p_{1} T+\alpha_{1} I_{H}+q_{1} D-r_{1}\right) \lambda_{1}+\lambda_{2} \alpha_{2} I_{H}-B_{1} \\
& \frac{d \lambda_{2}}{d t}=\left(\alpha_{2} T+d+q_{2} D-\beta I_{R}\right) \lambda_{2}+\lambda_{1} \alpha_{1} T+\lambda_{3} \beta I_{R}, \\
& \frac{d \lambda_{3}}{d t}=\left(2 r_{2} p_{2} I_{R}+\beta I_{H}+q_{3} D\right) \lambda_{3}-\lambda_{2} \beta I_{H}, \\
& \frac{d \lambda_{4}}{d t}=\lambda_{1} q_{1} T+\lambda_{2} q_{2} I_{H}+\lambda_{3} q_{3} I_{R}+\lambda_{4} \gamma,
\end{aligned}
$$

subject to the following conditions:

$$
\begin{gathered}
T(0)=T_{0}, \quad I_{H}(0)=I_{H_{0}}, \quad I_{R}(0)=I_{R_{0}} \\
D(0)=D_{0}, \\
\lambda_{i}\left(t_{f}\right)=0, \quad \text { for } i=1,2,3,4 .
\end{gathered}
$$

The previous analysis can be summarized in the following theorem.

Theorem 7. There exist an optimal control $u^{*}$ and corresponding solutions $T^{*}, I_{H}^{*}, I_{R}^{*}, D^{*}$ that minimize $J(u(t))$ over $U$. The explicit optimal controls are connected to the existence of continuous specific functions $\lambda_{i}(t), i=1,2,3,4$, the solutions of the following adjoint system:

$$
\begin{aligned}
& \frac{d \lambda_{1}}{d t}=\left(2 r_{1} p_{1} T+\alpha_{1} I_{H}+q_{1} D-r_{1}\right) \lambda_{1}+\lambda_{2} \alpha_{2} I_{H}-B_{1} \\
& \frac{d \lambda_{2}}{d t}=\left(\alpha_{2} T+d+q_{2} D-\beta I_{R}\right) \lambda_{2}+\lambda_{1} \alpha_{1} T+\lambda_{3} \beta I_{R} \\
& \frac{d \lambda_{3}}{d t}=\left(2 r_{2} p_{2} I_{R}+\beta I_{H}+q_{3} D\right) \lambda_{3}-\lambda_{2} \beta I_{H}, \\
& \frac{d \lambda_{4}}{d t}=\lambda_{1} q_{1} T+\lambda_{2} q_{2} I_{H}+\lambda_{3} q_{3} I_{R}+\lambda_{4} \gamma
\end{aligned}
$$

subject to the transversality conditions:

$$
\lambda_{i}\left(t_{f}\right)=0, \quad \text { for } i=1,2,3,4 \text {. }
$$

Furthermore, the following property holds:

$$
u^{*}=\min \left\{\max \left\{0,-\frac{\lambda_{4}}{B_{2}}\right\}, 1\right\} \text {. }
$$

\section{Numerical Simulations}

Analytical studies can never be completed without numerical verification of the derived results. In this section we present computer simulation of some important analytic results of our system discussed earlier. Beside verification of our analytical findings, these numerical simulations are very important from practical point of view.

The optimal system has been solved numerically and the results have been presented graphically. This optimal system is a two-point boundary value problem with separated boundary conditions at times $t=0$ and $t=t_{f}$. Here, we have solved this two-point boundary value optimality problem for $t_{f}=20$. The value is chosen to represent the time in days at which treatment is stopped. An efficient method to solve two-point BVPs numerically is collocation. A convenient collocation code is the solver BVP4c implemented under MATLAB, which can be used to solve nonlinear two-point BVPs. To solve our BVP we have used collocation method with collocation code solver BVP4c. It is a powerful method to solve the two-point BVP resulting from the optimality conditions.

The different variables (populations and control functions) in the objective functional given in (36) have different scales. Hence, they are balanced by choosing weight constants $B_{1}=5, B_{2}=10$ in the objective functional given in (36). The numerical results for the optimal problem are obtained by using the parameter values given in Table $2[2,33-36]$. At first we search for the optimal control function $u(t)$. This optimal control function $u(t)$ is designed in such a way that it minimizes the objective functional given by (36), that is, minimizes the number of tumor cells and the chemotherapeutic drug administration. In Figure 1 we have presented the time series diagrams of tumor cells, active CTL cells, and helper T-cells without any control $(u=0)$. In 
TABLE 2

\begin{tabular}{lc}
\hline Parameter & Value \\
\hline$r_{1}$ & $0.044 /$ day \\
$r_{2}$ & $0.0245 /$ day \\
$\alpha_{1}$ & $1.101 \times 10^{-7} /$ cells/day \\
$\alpha_{2}$ & $3.422 \times 10^{-10} /$ cells/day \\
$\beta$ & $6.2 \times 10^{-9} /$ cells/day \\
$\gamma$ & $0.01 /$ day \\
$p_{1}$ & $5 \times 10^{-9} /$ cells \\
$p_{2}$ & $1 \times 10^{-10} /$ cells \\
$q_{1}$ & $0.08 /$ day \\
$q_{2}$ & $2 \times 10^{-11} /$ day \\
$q_{3}$ & $1 \times 10^{-5} /$ day \\
$d$ & $0.0412 /$ day \\
\hline
\end{tabular}

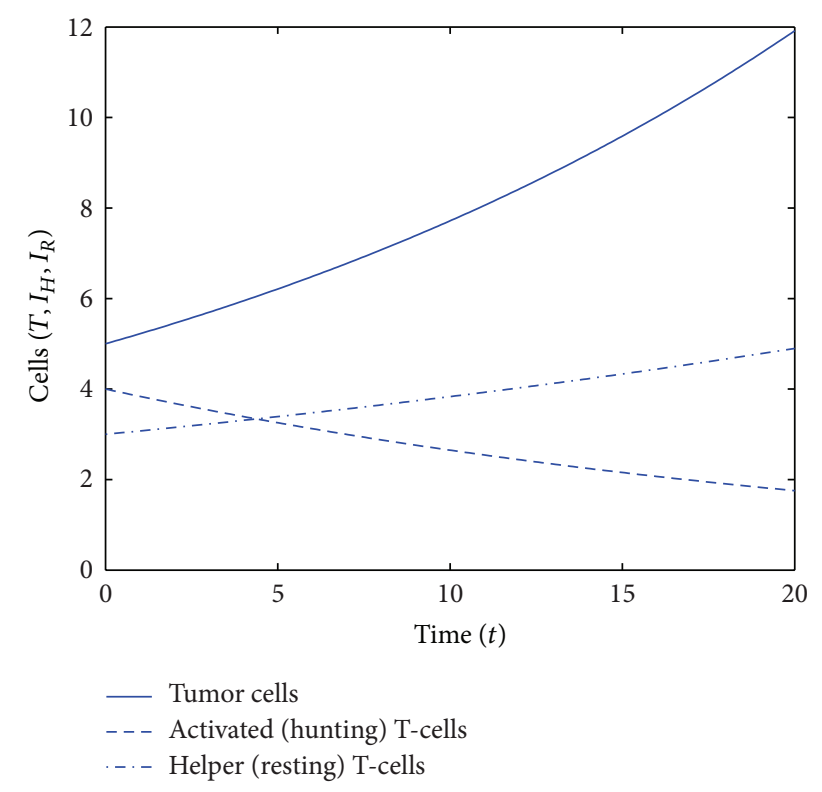

FIGURE 1: Time series plot of the tumor cell population $(T)$, active CTL cell population $\left(I_{H}\right)$, and helper T-cell population $\left(I_{R}\right)$ without control $(u=0)$ using the parameter values given in Table 2 with $B_{1}=5, B_{2}=10$.

Figure 2 we have presented the time series diagrams of tumor cells, active CTL cells, and helper T-cells with control $(u \neq 0)$.

As it is depicted in Figures 1 and 2, the tumor cell population $(T)$ level obtained using chemotherapeutic drug control is lower than its counterpart which results from practicing without control. From this observation we can conclude that the optimal control is much more effective for reducing the number of tumor cells to near zero. In perspective, one could conclude from the optimal control diagram (Figure 3 ) that we should give full effort in chemotherapeutic drug control in the beginning of the disease to reduce the spread of tumor cells. This means that chemotherapeutic drug is very much important in the beginning of the disease than when the disease prevails. From Figure 4 we observe that the chemotherapeutic drug control function $(u)$ minimizes the objective functional

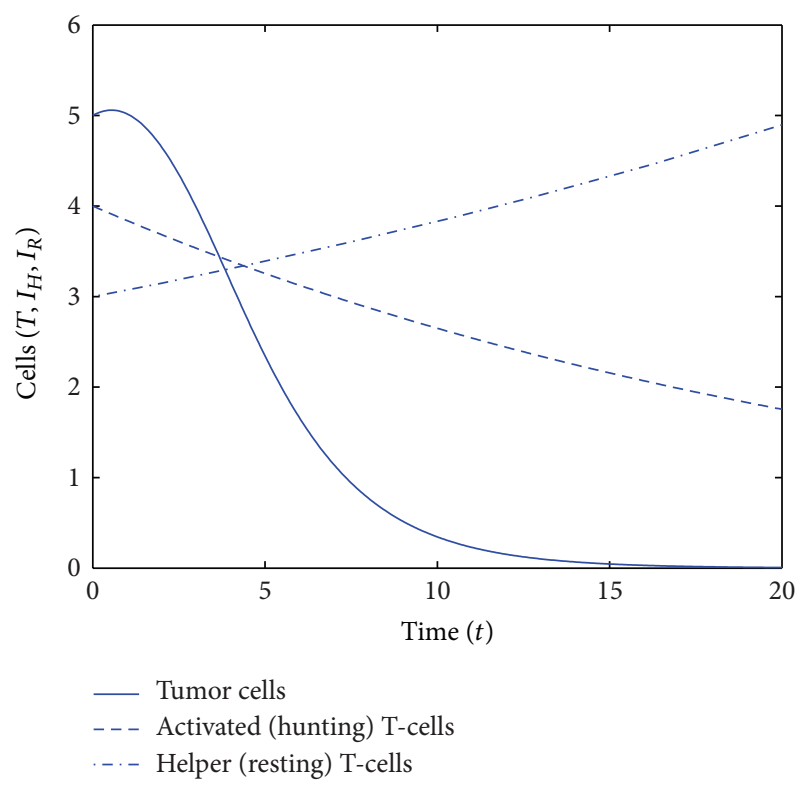

FIgURE 2: Time series plot of the tumor cell population $(T)$, active CTL cell population $\left(I_{H}\right)$, and helper T-cell population $\left(I_{R}\right)$ in presence of drug control $(u \neq 0)$ using the parameter values given in Table 2 with $B_{1}=5, B_{2}=10$.

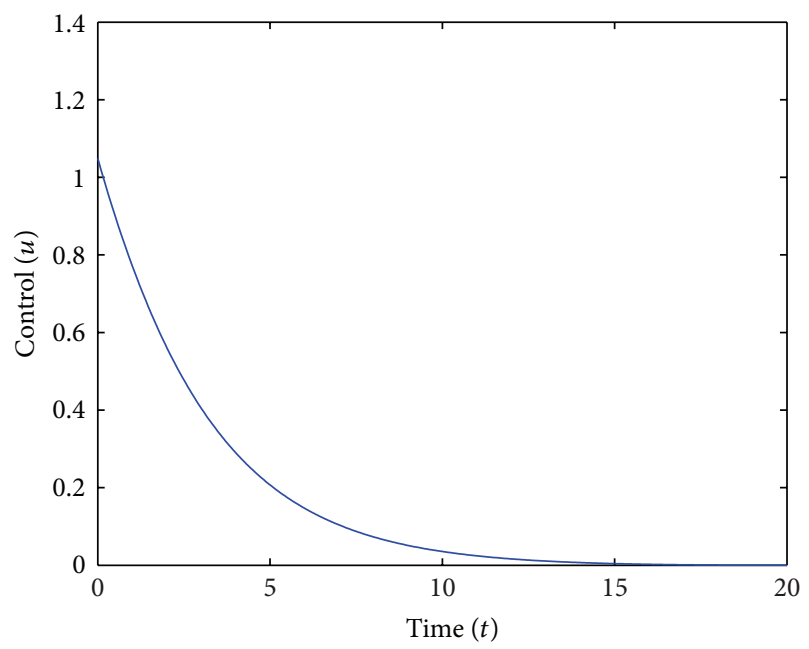

FIGURE 3: The optimal control graph for the chemotherapeutic drug control $(u)$ using the parameter values given in Table 2 with $B_{1}=5$, $B_{2}=10$.

given in (36). Overall the numerical analysis demonstrates that the control $u(t)$ decreases the tumor burden minimizing total drug administered. Numerical simulations also agreed with the theoretical characterization of the optimal control.

\section{Discussions and Conclusions}

In this paper, we have considered a malignant tumor growth model together with the effect of tumor-immune interaction and chemotherapeutic drug. Here we have explored the effects and interactions of tumor cells and CTL immune 


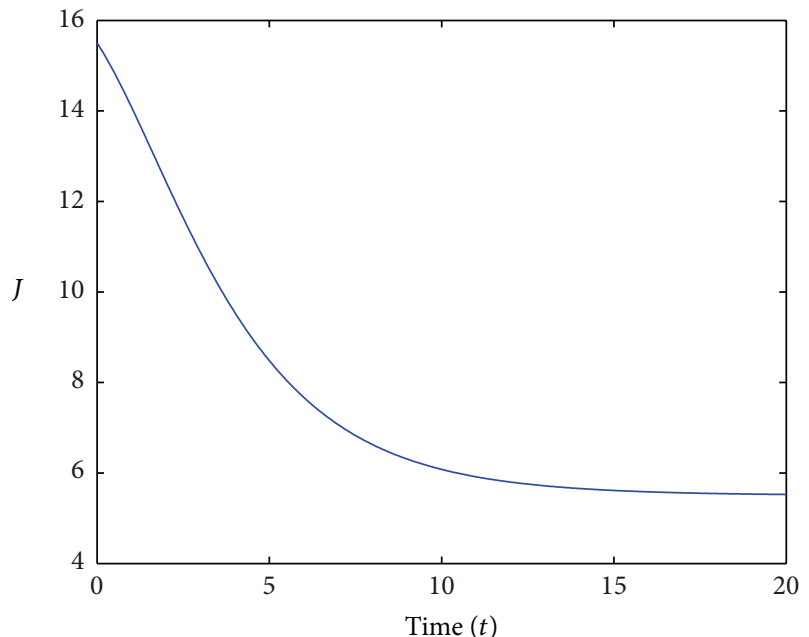

FIGURE 4: The optimal control graph for the objective functional $(J)$ using the parameter values given in Table 2 with $B_{1}=5, B_{2}=10$.

cells through a system of nonlinear differential equations. We have considered the effects of chemotherapeutic drug on the tumor cells as well as immune cells. Two types of CTL immune cells enter the model: (i) helper (resting) T-cells which are not able to attack and destroy tumor cells directly but release interleukin-2 which simulates CTLs and convert them into active (hunting) CTL cells and (ii) active (hunting) CTL cells which attack, destroy, or ingest the tumor cells. Next we have discussed dynamical behaviour of our system by analyzing the existence and stability of our system at various equilibrium points. For example, we have taken a set of estimated parameter values. Using them we have found that only one tumor free equilibrium becomes stable whereas the same equilibrium point becomes unstable without any chemotherapeutic drug. This sufficiently implies the necessity of using chemotherapeutic drug into the system.

The main focus of this paper is to set up an optimal control problem related to the model so as to minimize the number of tumor cells. We have considered the administration of chemotherapeutic drug as control to reduce the spread of the disease. Here we have used a quadratic control to quantify this goal. The quadratic control reflects the severity of the side effects of the drug imposed $[29,30]$. When chemotherapeutic drugs are administered in high dose, they are toxic to the human body, which justifies the use of quadratic control. The control function $u(t)$ is designed in such a way that minimizes the objective functional or cost function as given in (36).

The important mathematical findings for the dynamical behaviour of the tumor-immune model with control are also numerically verified using MATLAB. The graphical representations of the model with control as well as without control are presented for tumor cells and two types of immune cells so that we can compare them and can understand the effectiveness of using the control. It is observed that the optimal control is much more effective for reducing the number of tumor cells to near zero. Overall the numerical analysis demonstrates that a burst of treatment at the beginning is the best way to fight against the tumor cells. Numerical simulations agreed with the theoretical characterization of the optimal control.

The mathematical models on diseases are rather simple, but, nevertheless, they give insight into some of the consequences of public health policies. Our model formulation is based on the effects and interactions of tumor cells and immune cells and also the effects of chemotherapeutic drug on both tumor cells and CTL immune cells. We have also considered a model with control where the administration of chemotherapeutic drug is treated as control. Our model can provide an approximate estimation of timing and dosage of therapy that would be the best complement of the patient's own defense mechanism versus the tumor cells. As with many models, the mathematical model presented in this paper should be treated with circumspection due to the assumptions made and the difficulties in the estimation of the model parameters. Most of the parameters are dependent on many factors, so they are rarely constants. But for the simplification of the system, these parameters are taken as constants. The activation of CTL cells, attacking and destroying tumor cells, is not instantaneous; rather there may be some time lags. Therefore as a part of the future work the model considered here can be refined to incorporate time delays in the system to make it more realistic. Also, there are many components in this model that may be regarded as stochastic rather than deterministic, and these variations may significantly alter the dynamics of the system. Therefore, as our future work we can incorporate stochastic differential equations in modelling and study its dynamics. Controlling the spread of tumor cells is now a challenging and important issue to study. Chemotherapy, immunotherapy, radiation therapy, surgery, and so forth are most useful therapies to control and reduce the spread of tumor cells. So, the development of these therapies and identification of the most effective therapy against the spread of tumor cells are the primary goal of health administrators, policy-makers, and researchers. Our model study is a small step towards the goal by which we want to identify the parameters of interest for further study.

\section{Acknowledgments}

The authors are grateful to the anonymous referees and the editor (Professor Giovanni P. Galdi) for their careful reading, valuable comments, and helpful suggestions, which have helped them to improve the presentation of this work significantly.

\section{References}

[1] V. A. Kuznetsov and G. D. Knott, "Modeling tumor regrowth and immunotherapy," Mathematical and Computer Modelling, vol. 33, no. 12-13, pp. 1275-1287, 2001.

[2] V. A. Kuznetsov, I. A. Makalkin, M. A. Taylor, and A. S. Perelson, "Nonlinear dynamics of immunogenic tumors: parameter estimation and global bifurcation analysis," Bulletin of Mathematical Biology, vol. 56, no. 2, pp. 295-321, 1994. 
[3] D. Kirschner and J. C. Panetta, "Modeling immunotherapy of the tumor-immune interaction," Journal of Mathematical Biology, vol. 37, no. 3, pp. 235-252, 1998.

[4] M. Kolev, "Mathematical modelling of the competition between tumors and immune system considering the role of the antibodies," Mathematical and Computer Modelling, vol. 37, no. 11, pp. 1143-1152, 2003.

[5] L. G. De Pillis, W. Gu, and A. E. Radunskaya, "Mixed immunotherapy and chemotherapy of tumors: modeling, applications and biological interpretations," Journal of Theoretical Biology, vol. 238, no. 4, pp. 841-862, 2006.

[6] L. G. De Pillis and A. Radunskaya, "A mathematical tumor model with immune resistance and drug therapy: an optimal control approach," Journal of Theoretical Medicine, vol. 3, no. 2, pp. 79-100, 2001.

[7] J. C. Arciero, T. L. Jackson, and D. E. Kirschner, "A mathematical model of tumor-immune evasion and siRNA treatment," Discrete and Continuous Dynamical Systems B, vol. 4, no. 1, pp. 3958, 2004.

[8] N. Bellomo, A. Bellouquid, and M. Delitala, "Mathematical topics on the modelling complex multicellular systems and tumor immune cells competition," Mathematical Models and Methods in Applied Sciences, vol. 14, no. 11, pp. 1683-1733, 2004.

[9] N. Bellomo and L. Preziosi, "Modelling and mathematical problems related to tumor evolution and its interaction with the immune system," Mathematical and Computer Modelling, vol. 32, no. 3-4, pp. 413-452, 2000.

[10] B. S. Chan and P. Yu, "Bifurcation analysis in a model of cytotoxic T-lymphocyte response to viral infections," Nonlinear Analysis: Real World Applications, vol. 13, no. 1, pp. 64-77, 2012.

[11] L. G. De Pillis, A. E. Radunskaya, and C. L. Wiseman, "A validated mathematical model of cell-mediated immune response to tumor growth," Cancer Research, vol. 65, no. 17, pp. 7950$7958,2005$.

[12] L. Derbel, "Analysis of a new model for tumor-immune system competition including long-time scale effects," Mathematical Models and Methods in Applied Sciences, vol. 14, no. 11, pp. 16571681, 2004.

[13] A. D’Onofrio, “A general framework for modeling tumorimmune system competition and immunotherapy: mathematical analysis and biomedical inferences," Physica D, vol. 208, no. 3-4, pp. 220-235, 2005.

[14] F. Nani and H. I. Freedman, "A mathematical model of cancer treatment by immunotherapy," Mathematical Biosciences, vol. 163, no. 2, pp. 159-199, 2000.

[15] S. T. R. Pinho, F. S. Bacelar, R. F. S. Andrade, and H. I. Freedman, "A mathematical model for the effect of anti-angiogenic therapy in the treatment of cancer tumors by chemotherapy," Nonlinear Analysis: Real World Applications, vol. 14, pp. 815-828, 2013.

[16] H. Siu, E. S. Vivetta, R. D. May, and J. W. Uhr, "Tumor dormancy-I. Regression of BCL1 tumor and induction of a dormant tumor state in mice chimeric at the major histocompatibility complex," Journal of Immunology, vol. 137, no. 4, pp. 1376-1382, 1986.

[17] T. Takayanagi and A. Ohuchi, "A mathematical analysis of the interactions between immunogenic tumor cells and cytotoxic $\mathrm{T}$ lymphocytes," Microbiology and Immunology, vol. 45, no. 10, pp. 709-715, 2001.

[18] R. Yafia, "Dynamics analysis and limit cycle in a delayed model for tumor growth with quiescene," Nonlinear Analysis. Modelling and Control, vol. 11, pp. 95-110, 2006.
[19] R. Martin and K. L. Teo, Optimal Control of Drug Administration in Cancer Chemotherapy, World Scientific, River Edge, NJ, USA, 1994.

[20] A. S. Matveev and A. V. Savkin, "Application of optimal control theory to analysis of cancer chemotherapy regimens," Systems and Control Letters, vol. 46, no. 5, pp. 311-321, 2002.

[21] L. G. de Pillis, W. Gu, K. R. Fister et al., "Chemotherapy for tumors: an analysis of the dynamics and a study of quadratic and linear optimal controls," Mathematical Biosciences, vol. 209, no. 1, pp. 292-315, 2007.

[22] L. G. De Pillis and A. Radunskaya, "The dynamics of an optimally controlled tumor model: a case study," Mathematical and Computer Modelling, vol. 37, no. 11, pp. 1221-1244, 2003.

[23] M. Engelhart, D. Lebiedz, and S. Sager, "Optimal control for selected cancer chemotherapy ODE models: a view on the potential of optimal schedules and choice of objective function," Mathematical Biosciences, vol. 229, no. 1, pp. 123-134, 2011.

[24] K. R. Fister and J. Donnelly, "Immunotherapy: an optimal control theory approach," Mathematical Biosciences and Engineering, vol. 2, no. 3, p. 499, 2005.

[25] K. R. Fister and J. C. Panetta, "Optimal control applied to cellcycle-specific cancer chemotherapy," SIAM Journal on Applied Mathematics, vol. 60, no. 3, pp. 1059-1072, 2000.

[26] K. R. Fister and J. C. Panetta, "Optimal control applied to competing chemotherapeutic cell-kill strategies," SIAM Journal on Applied Mathematics, vol. 63, no. 6, pp. 1954-1971, 2003.

[27] U. Ledzewicz and H. Schättler, "Antiangiogenic therapy in cancer treatment as an optimal control problem," SIAM Journal on Control and Optimization, vol. 46, no. 3, pp. 1052-1079, 2007.

[28] S. M. Mamat and A. Kartono, "Mathematical model of cancer treatment using immunotherapy, chemotherapy and biochemotherapy," Applied Mathematical Sciences, vol. 7, no. 5, pp. 247261, 2013

[29] H. R. Joshi, "Optimal control of an HIV immunology model," Optimal Control Applications and Methods, vol. 23, no. 4, pp. 199-213, 2002.

[30] G. Zaman, Y. Han Kang, and I. H. Jung, "Stability analysis and optimal vaccination of an SIR epidemic model," BioSystems, vol. 93 , no. 3, pp. 240-249, 2008.

[31] J. K. Hale, Theory of Functional Differential Equations, Springer, New York, Ny, USA, 1977.

[32] M. Kot, Elements of Mathematical Ecology, Cambridge University Press, Cambridge, UK, 2001.

[33] L. Bannock, http://www.doctorbannock.com/nutrition.html.

[34] P. Calabresi and P. S. Schein, Eds., Medical Oncology: Basic Principles and Clinical Management of Cancer, McGraw-Hill, New York, NY, USA, 2nd edition, 1993.

[35] A. Diefenbach, E. R. Jensen, A. M. Jamieson, and D. H. Raulet, "Rael and H60 ligands of the NKG2D receptor stimulate tumour immunity," Nature, vol. 413, no. 6852, pp. 165-171, 2001.

[36] M. C. Perry, Ed., The Chemotherapy Source Book, Lippincott Williams and Wilkins, 3rd edition, 2001.

[37] K. Blayneh, Y. Cao, and H.-D. Kwon, "Optimal control of vectorborne diseases: treatment and prevention," Discrete and Continuous Dynamical Systems, vol. 11, no. 3, pp. 587-611, 2009.

[38] S. Lcnhart and J. T. Workman, Optimal Control Applied to Biological Mathods, Chapman and Hall/CRC, London, UK, 2007.

[39] G. W. Swan, Applications of Optimal Control Theory in Biomedicine, Marcel Dekker, New York, NY, USA, 1984. 
[40] J. M. Tchuenche, S. A. Khamis, F. B. Agusto, and S. C. Mpeshe, "Optimal control and sensitivity analysis of an influenza model with treatment and vaccination," Acta Biotheoretica, vol. 59, no. 1, pp. 1-28, 2011.

[41] W. H. Fleming and R. W. Rishel, Deterministic and Stochastic Optimal Control, Springer, New York, NY, USA, 1975.

[42] D. L. Lukes, Differential Equations: Classical to Controlled, Mathematics in Science and Engineering, Academic Press, New York, NY, USA, 1982.

[43] L. S. Pontryagin, V. G. Boltyanskii, R. V. Gamkrelidze, and E. F. Mishchenko, The Mathematical Theory of Optimal Process, Gordon and Breach, 1962. 

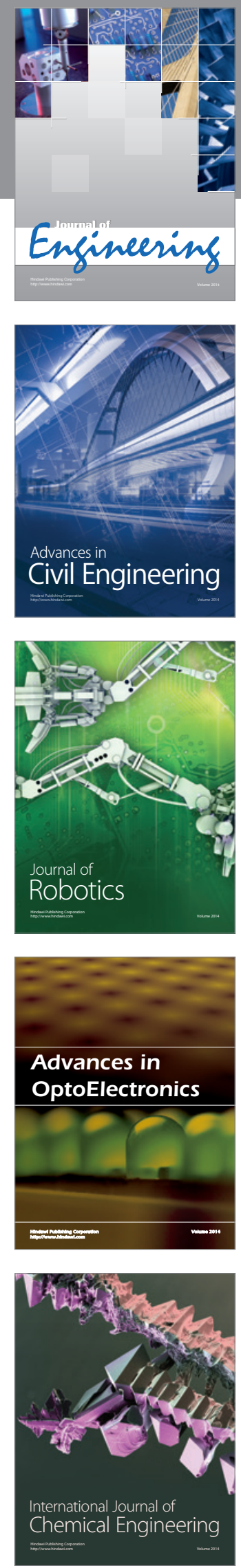

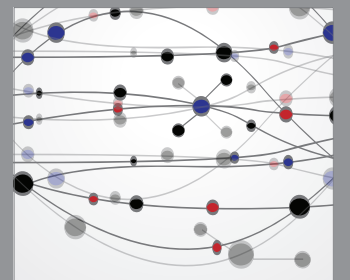

The Scientific World Journal
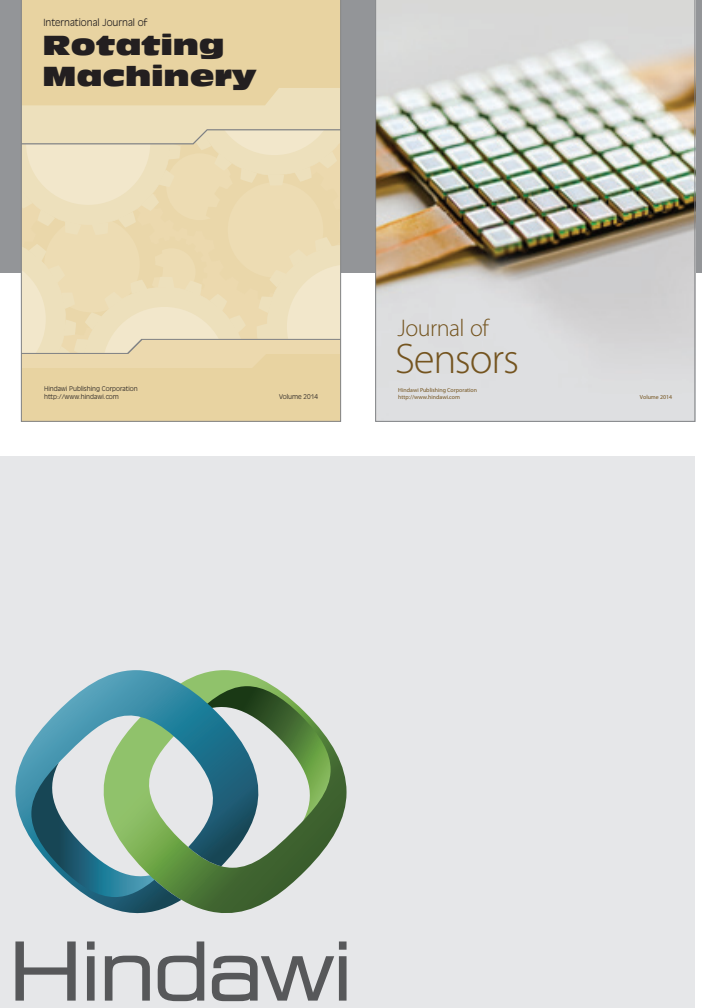

Submit your manuscripts at http://www.hindawi.com
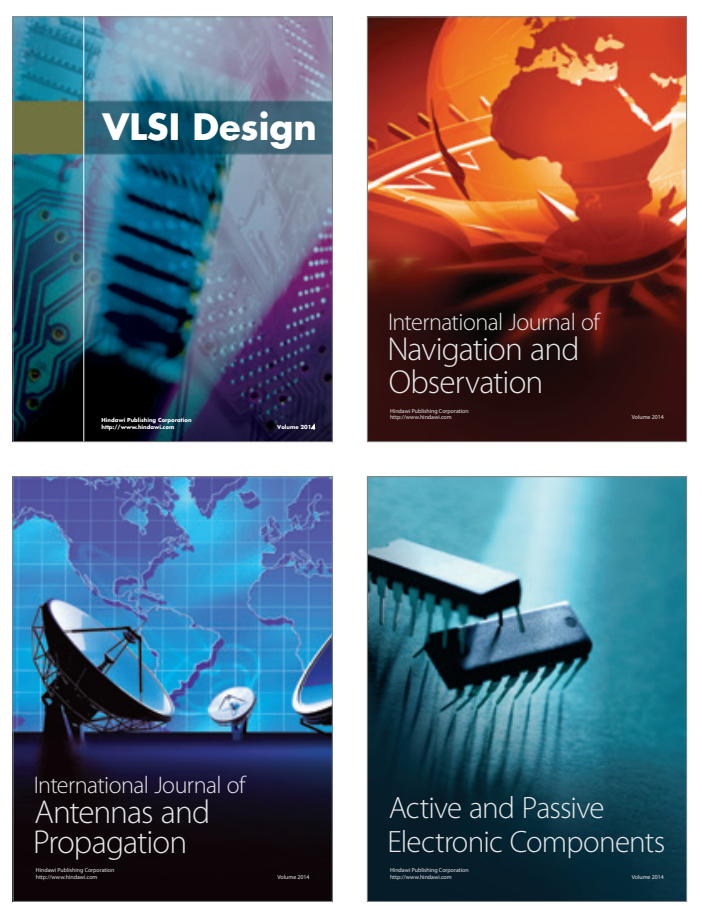
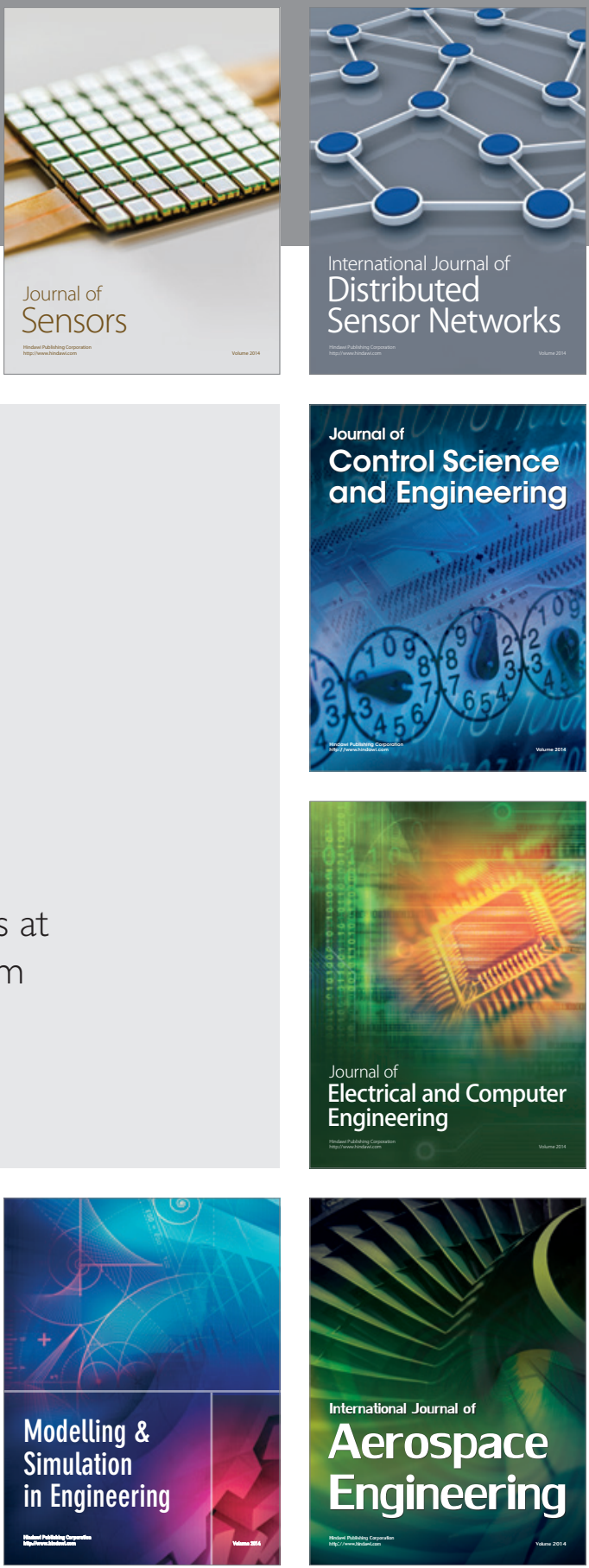

Journal of

Control Science

and Engineering
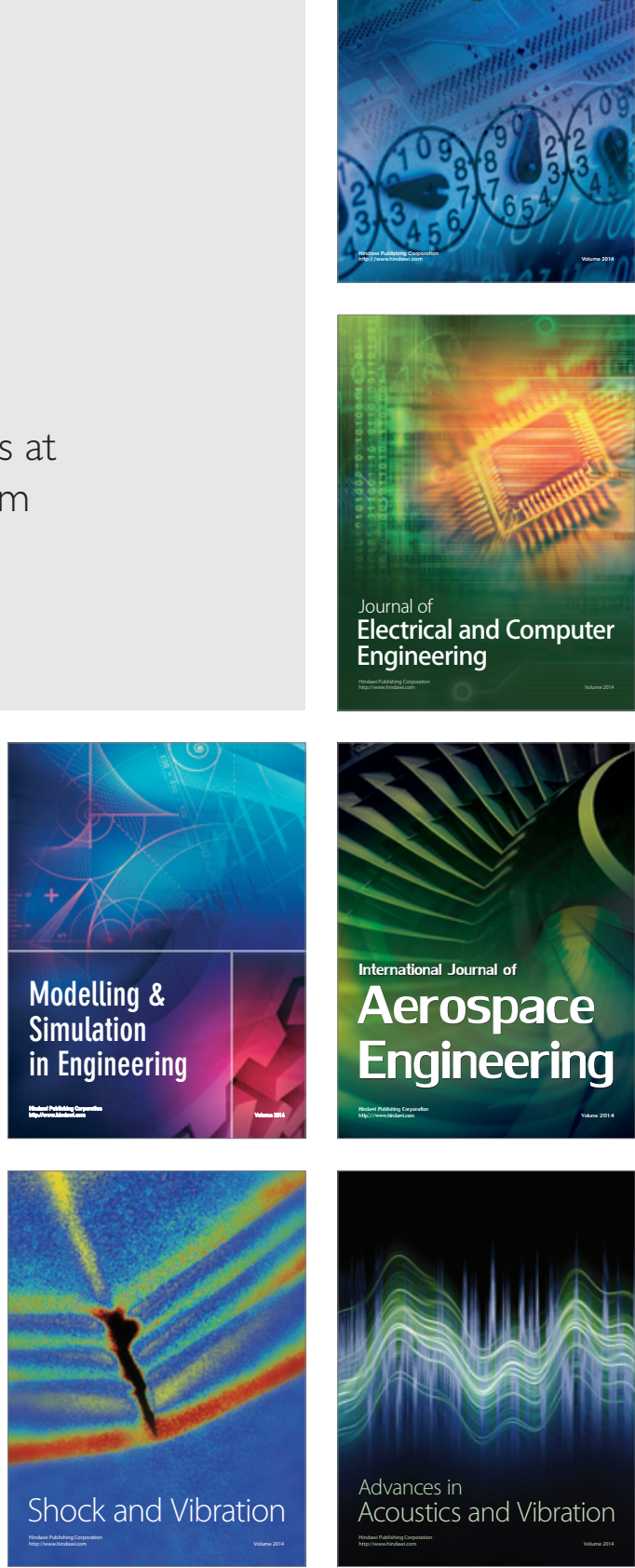